\title{
Smell and taste recognition in early stages of late-onset Alzheimer's disease
}

\author{
Nila Ilhamto, Lisa Duizer* \\ *This study was conducted under the supervision of Professor Duizer, \\ Department of Food Science, Ontario Agricultural College, \\ University of Guelph, Guelph, Ontario, Canada
}

\begin{abstract}
Problems of inadequate nutrition and energy intake are common in the aging population. Smell and taste deficits associated with Late-Onset Alzheimer's Disease (LOAD) may accentuate the decline in nutritional status of elderly individuals and indirectly enhance progression of cognitive problems in LOAD. The objective of this study was to explore and characterize smell and taste recognition abilities in early stages of LOAD, beyond that of normal healthy aging. A total of 29 healthy-younger subjects aged 18-40 (HY), 13 healthy-elderly (HA) and six elderly adults diagnosed with LOAD (AD) aged 60-85, were recruited from the Guelph community. The Sniffin' Sticks Screening Test (SSST) and Taste Strips were used to test olfactory and gustatory functions, respectively. Participants also completed the mini-mental state examination (MMSE), clock test and word recall tests to assess cognitive/memory skills. Compared to HA individuals, people with $\mathrm{AD}$ had significant odour recognition impairment. Correlation analysis also revealed an age-associated decline in overall taste ability. When specific tastes were examined, impairments in sour and bitter identification were observed with increasing age. However, no significant differences in specific taste abilities were found between HA and AD individuals. In predicting health status (ie. presence or absence of LOAD), an assessment of all variables in this study was conducted using Generalized Linear Model (GLM). Results showed that sweet recognition and clock test scores were the best predictive variables of health status. However, this is a preliminary model that needs refinement through further research using more individuals.
\end{abstract}

$\mathrm{L}$ ate-Onset Alzheimer's disease (LOAD) is a progressive neurodegenerative disease affecting elderly individuals aged 60 and older [1]. Deficits in odour identification are apparent in early, pre-symptomatic stages of LOAD, while odour sensitivity decline seems to occur in later stages of the disease. The decline in olfactory identification may be a reflection of neuropathology that is the LOAD hallmark. Some odour information from the olfactory bulb is received in the entorhinal cortex which is also the location of the first appearance of neuritic plaques (NPs) and neurofibrillary tangles (NFTs) prevalent in individuals with LOAD [2,3]. It is the accumulation of these plaques and tangles, in the early pre-clinical stages of LOAD that may be responsible for disrupting essential olfactory information processing needed to identify odours.

There have been studies to suggest that, like odour identification, taste identification is also impaired in the early-stages of LOAD [4]. This may be due to changes in the innervation of the taste buds. After ingestion of a tastant, taste buds are activated and gustatory information is carried by the facial (VII), glossopharyngeal (IX) and vagus (X) nerves to nuclear regions of the brain for processing [5]. Specific studies on patients with LOAD reveal significant declines in the innervation of taste buds located in the foliate and circumvallate papillae [6]. As well, more recent studies at the level of the taste bud demonstrate that brain derived neurotrophic factor (BDNF) is essential in the development of fungiform papillae taste buds [7]. In LOAD, the presence of NPs attenuates the transcription of BDNF in the hippocampus, thereby altering taste sensations [7].

Research in senile-dementia cases (not exclusive to LOAD) illustrated significant losses in the ability to identify the four basic tastes (sour, sweet, salty and bitter) [8,9]. Significant impairments in overall taste and individual basic tastes have also recently been found in individuals with LOAD in comparison to individuals with mild cognitive impairment (MCI) and age-matched controls [10]. However, a comparison of taste abilities with younger adults has not been conducted. It has been estimated that for every 100,000 persons at risk for Alzheimer's disease, the prevalence of developing Alzheimer's disease in individuals under the age of 60 is less than $0.5 \%$ [11]. Because the incidence of developing Alzheimer's is rare in younger adults, we have assumed that the population of younger adults recruited for the study did not have Alzheimer's and were a healthy control population. Therefore, the objective of this study was to investigate smell and taste function in early-stages of 
LOAD, in comparison to elderly and younger healthy controls.

\section{METHODS}

\section{Participants and recruitment}

A total of 48 volunteers were recruited from the community of Guelph through print media: six individuals, aged 60-85, clinically diagnosed with LOAD by a certified physician within the last five years (labelled as $\mathrm{AD}$ in the remainder of this paper), 13 healthy-aging individuals, aged 60-85, without LOAD or any other dementia-type diseases (labelled as HA in the remainder of this paper) and 29 healthy-younger adults, aged 18-40, without $\mathrm{AD}$ (labelled as $\mathrm{HY}$ in the remainder of this paper).

\section{Materials and methods}

This study was reviewed and received clearance from the University of Guelph Research Ethics Board (REB\#09OC022). Participants completed a demographic questionnaire and a detailed health screening form. Questions regarding age, gender, highest education level and number of pharmaceutical drugs taken were asked. Volunteers allergic or highly sensitive to substances in the experimental design were excluded. Those with any other dementia-related diseases or relevant co-morbidities that may be affecting smell and taste were also excluded. Participants or their Power of Attorneys' (POAs) signed a written consent form indicating their voluntary participation in the study.

Neuropsychological tests were administered to assess attention, memory and cognitive skills. The mini-mental state examination (MMSE) is a classic neuropsychological test, widely used to detect cognitive impairment [12]. During the MMSE test, participants were asked a series of 11 questions to assess a range of cognitive domains including: orientation, registration, attention, recall and language.

To further supplement the MMSE, the Clock-Drawing Test [13] and the immediate and delayed word recall tests from the Consortium to Establish a Registry for Alzheimer's Disease (CERAD) [14], were administered. The clock test followed the free-drawing Freedman method [15]. Participants were given a blank piece of paper and a pen. They were asked to "draw the face of a clock with all of the numbers in it and the hands of the clock displaying 2:45". The CERAD immediate and delayed word recall test consisted of a list of ten, unrelated words. Participants repeated aloud ten words, one at a time after the administrator. Each word was presented to the participant every 2-3 seconds. After repetition of each word, they were instructed to recall as many of the ten words as they could remember, with no preference as to the order they were recalled. This task was repeated a total of three times. After the third time, there was a distraction period of 10 minutes where participants drew shapes, made lists and computed arithmetic questions before they were asked to verbally recall any of the ten words again for a 4 th trial (delayed recall).
Following the neuropsychological tests, the smell and taste tests were administered to each participant individually. To assess olfactory identification abilities, participants were subjected to a brief olfactory test, called the Sniffin' Sticks Screening Test (SSST) (Burghart Medizintechnik, Wedel, Germany). The test contained 12 pen-like devices with felt tips encapsulated with different odours $(4 \mathrm{~mL}$ odorant dissolved in propylene glycol). Volunteers sniffed each penlike device in a natural manner, placing the stick approximately $2 \mathrm{~cm}$ under the nose. They then identified the smell from a list of four written options. Each written option was also accompanied by a picture of that option. To reset olfactory receptor neurons (ORNs), there was a waiting period of 30 seconds in between each stick. For all 12 sticks administered, participants sniffed alternatively under the right and left nose. To assess taste abilities, Taste Strips were used (Burghart Medizintechnik, Wedel, Germany). Each taste strip was comprised of a strip of filter paper with a $6 \mathrm{~cm}$ long handle and a $2 \mathrm{~cm} 2$ tip area impregnated with different concentrations of bitter, salty, sweet or sour taste solutions. Four concentrations for each of the basic tastes were administered: sweet $(0.4,0.2,0.1,0.05 \mathrm{~g} / \mathrm{mL}$ sucrose $)$, sour $(0.3,0.165,0.09,0.05 \mathrm{~g} / \mathrm{mL}$ citric acid), salty $(0.25,0.1$, $0.04,0.016 \mathrm{~g} / \mathrm{mL} \mathrm{NaCl})$, bitter $(0.006,0.0024,0.0009$, $0.0004 \mathrm{~g} / \mathrm{mL}$ quinine hydrochloride). Four blank strips were also included in this study to provide a no taste option to the participants. Each strip was placed on the right or left anterior one third of the extended tongue, alternatively. The strips were rubbed 3-4 times on the lateral, medial and tip of the tongue. Participants indicated whether the strip was sweet, salty, sour, bitter or had no taste. The written options "sweet", "salty", "sour" "bitter" and "no taste" with visual aids served as examples to assist in making the decision. In between each strip, volunteers sipped water to cleanse their palate and wash out any residual taste from the previous strips.

Twenty taste strips were evaluated by each person in a series of four blocks of five strips. The five strips were the four tastants plus the blank. Presentation of the strips was randomized within each block. The first block of five strips administered to the participants contained the lowest concentrations of sweet, sour, salty, bitter and a blank strip. The next five strips administered, were at concentrations higher than the first block. Presentations of the blocks continued until the maximum intensity of tastants were administered.

\section{Analysis}

For analysis, responses to demographic questions were collated to determine the ages and the number of males and females completing the test. The highest education level completed was allocated numbers such that: $0=$ no schooling, $1=$ grade school, $2=$ high school, $3=$ college/undergraduate, $4=$ graduate. The number of pharmaceutical drugs consumed by participants was also determined. 
Table 1: Demographic data displaying means and standard deviations (sd) for healthy young (HY), healthy aging (HA) and late-onset Alzheimer disease (AD) groups.

\begin{tabular}{|l|r|r|r|r|r|r|}
\hline \multirow{2}{*}{} & \multicolumn{2}{|c|}{$H Y$} & \multicolumn{2}{c|}{$H A$} & \multicolumn{2}{|c|}{$A D$} \\
\cline { 2 - 7 } & \multicolumn{1}{|c|}{ Mean } & sd & Mean & sd & Mean & sd \\
\hline Mean age (yrs) & 22.14 & 3.40 & 74.77 & 6.65 & 77.50 & 4.76 \\
\hline Women (\%) & 68.97 & 0.47 & 69.23 & 0.48 & 33.33 & 0.52 \\
\hline Highest Education Level & 3.03 & 0.19 & 2.54 & 0.88 & 3.00 & 0.89 \\
\hline Medication counts & 0.28 & 0.84 & 2.46 & 1.85 & 3.33 & 3.88 \\
\hline
\end{tabular}

Data collected from the MMSE test, clock test and word recall tests were used to determine cognitive skills. The MMSE was scored out of 30, with points allocated for correct responses. The clock drawing test was scored using the free-drawing Freedman method, which uses a 15-point scoring system; six points were given for numbers and hands, two points to contour, and one to the center [15]. The CERAD immediate recall score was the sum of three trials, out of 30 , while delayed recall consisted of one trial out of 10.

Counts of correct responses from the smell test (scored out of 12), overall taste test (scored out of 16) and for the individual basic tastes (each scored out of four) were made and these counts were used in all data analysis.

Statistical analyses of all of the data were done in "R" foundation of Statistical Computing, version 2.10.1., Copyright 2009. Means and standard deviations were tallied and tabulated for participant demographic profiles (age, gender, highest education completed and number of pharmaceutical drugs taken). A Kruskal-Wallis rank sums test was conducted to determine if significant differences in demographics and smell and taste recognition abilities existed among the three groups (HY, HA and AD). A posthoc analysis was performed using Multiple Comparisons after Kruskal-Wallis. For comparing age in the three groups, a one-way ANOVA and a post-hoc Tukey's HSD test was conducted. Correlation analysis to determine the association between neuropsychological and demographic factors with the smell and taste recognition tests was conducted using Spearman's. To find the subset of variables highly influencing health status (ie. presence or absence of LOAD) in this study, a step-wise multiple regression analysis called the Generalized Linear Model (GLM) was used. GLM is a linear model used to fit a line to data that do not exclusively follow a normal distribution [16]. The process automatically predicts which variables to add and drop based on the proportion of variance it has upon health status. The Akaike Information Criterion (AIC), as shown in Table 6, is the method of selecting a model from a set of models. The final linear model selected should be the closest fit from the truth, except for a few parameters [17]. The estimates (b coefficients and constant) can be used to construct a prediction equation and generate predicted health status for further analysis.
Table 2: Correlation coefficient $(R)$ values determined using the Spearman's method for smell and taste recognition abilities, with demographics and other external factors as independent factors.

\begin{tabular}{|l|l|l|l|l|}
\hline & $\begin{array}{l}\text { Highest } \\
\text { Education }\end{array}$ & Age & Sex & Medication \\
\hline Smell test & 0.0817 & -0.2840 & 0.1928 & -0.2176 \\
\hline Overall taste & 0.2631 & $-0.5537^{* * *}$ & 0.2278 & $-0.3298^{*}$ \\
\hline Sweet & $0.4001^{* *}$ & -0.1538 & 0.0640 & -0.2712 \\
\hline Salty & 0.0838 & -0.2416 & 0.0802 & -0.1040 \\
\hline Sour & 0.1761013 & $-0.5706^{* * *}$ & 0.2141 & -0.2512 \\
\hline Bitter & 0.1427 & $-0.5057^{* * *}$ & $0.3174^{*}$ & $-0.3001^{*}$ \\
${ }^{*} p<0.05,{ }^{* *} p<0.01,{ }^{* * *} p<0.001$ &
\end{tabular}

\section{RESULTS AND DISCUSSION}

The aim of this study was to examine differences in chemosensory deficits experienced in normal aging and in LOAD. For this purpose, we recruited $\mathrm{AD}, \mathrm{HA}$ and $\mathrm{HY}$ individuals. Age, sex, education level and the number of medications taken by each participant were recorded, and means and standard deviations were tabulated in Table 1. The demographic profile in Table 1 shows that healthy aging and $\mathrm{AD}$ groups were matched for age $(\mathrm{p}=0.46)$. All three groups were also matched for percentage of women $(\mathrm{p}=$ $0.24)$ and for highest achieved education level $(p=0.074)$. HY individuals take significantly less medications than HA or $\mathrm{AD}$ groups $(\mathrm{p}=8.59 \times 10-6)$.

The demographic factors in Table 1 (age, sex, education level and medication) were correlated with smell and taste abilities (Table 2). Overall taste identification as well as sour and bitter identification decreased as age increased, as shown by the significant negative correlations in Table 2. There are many reasons why taste dysfunctions occur, including situations such as tooth loss, upper respiratory infections, head injury and decreased saliva production [18]. The combination of increasing age and medication intake may be the most debilitating contributor of taste disorders in the elderly, particularly when multiple pharmaceutical drugs are being consumed [19]. This is confirmed from the results of this study, where a high medication count was related to poor overall tasting and bitter recognition performance (Table 2). It has been estimated that people over the age of 65 only comprise $13 \%$ of the population, yet they consume about one-third of all prescribed medications [19] and almost all elderly individuals take multiple pharmaceutical drugs. This is confirmed in the current study. All of the elderly adults (AD and HA) consumed multiple drugs (mean counts of 3.33 and 2.46, respectively; Table 1).

The aptitude to identify sweet and salty were not significantly affected by age in this study while sour and bitter were (Table 2). Although females had higher mean performance scores in both smell and taste identification tests only bitter identification was significantly different between males and females $(p=0.037)$. Table 2 shows a significant correlation between women and higher bitter recognition 
Table 3: Means (m) and standard deviations (sd) for smell and taste recognition abilities were determined for healthyyoung (HY), healthy-aging (HA) and Alzheimer disease (AD) groups.

\begin{tabular}{|l|r|r|r|r|r|r|}
\hline & \multicolumn{2}{|c|}{$H Y$} & \multicolumn{2}{c|}{$H A$} & \multicolumn{2}{|c|}{$A D$} \\
\hline & $\mathrm{m}$ & $\mathrm{sd}$ & $\mathrm{m}$ & $\mathrm{sd}$ & $\mathrm{M}$ & $\mathrm{sd}$ \\
\hline Smell test & 10.41 & 1.18 & 10.15 & 1.67 & 5.00 & 3.22 \\
\hline $\begin{array}{l}\text { Overall } \\
\text { taste }\end{array}$ & 12.79 & 2.16 & 10.08 & 2.66 & 6.67 & 2.16 \\
\hline Sweet & 3.72 & 0.53 & 3.31 & 1.03 & 2.33 & 1.63 \\
\hline Salty & 3.03 & 0.82 & 2.77 & 1.17 & 2.17 & 0.41 \\
\hline Sour & 2.86 & 0.83 & 1.62 & 1.19 & 0.5 & 0.55 \\
\hline Bitter & 3.17 & 1.04 & 2.38 & 1.12 & 1.67 & 0.82 \\
\hline
\end{tabular}

abilities. It is well documented that women are more likely to be supertasters of bitter substances when compared to their male counterparts $[20,21]$ possibly due to their anatomy. Not only do women have more fungiform papillae and taste buds than men, they also have concentration differences of cAMP and cGMP in nasal mucus [22] and parotid saliva [23] possibly leading to gender differences in smell and taste function.

\section{Smell and overall taste function}

Mean smell and taste recognition abilities are shown in Table 3 and the median and spread of smell identification scores in all three groups (HY, HA and AD) are shown in Figure 1a). Healthy-younger (HY) and healthy-aging (HA) groups identified odours significantly better than AD individuals $(p=0.0027$ and $p=0.012$ for HY and HA, respectively) (Table 4). Results from Table 4 also confirm previous research showing greater decrements in the ability to identify odours in early stages of LOAD than in HA individuals $[3,24,25,26]$. These findings are supported by anatomical characteristics of LOAD pathology. In presymptomatic stages of LOAD, one area of the brain where NPs and NFTs are first deposited is in the entorhinal cortex. This area of the brain has been shown to be involved in

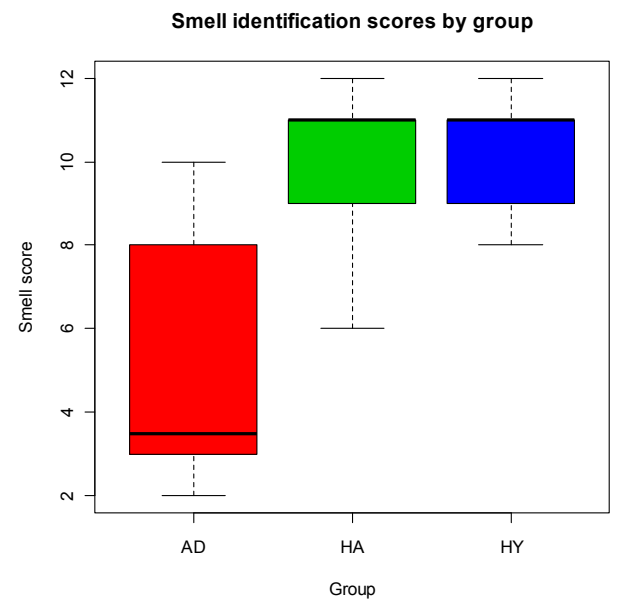

Table 4: Smell and taste test P-values (P) for two-group comparisons (HY-HA, HY-AD, HA-AD). "TRUE" represents $P<0.05$ and "FALSE" represents $P>0.05$.

\begin{tabular}{|l|l|l|l|}
\hline \multicolumn{1}{|c|}{$H$ HY-HA } & \multicolumn{1}{c|}{$H Y-A D$} & \multicolumn{1}{c|}{$H A-A D$} \\
\hline Smell Test & FALSE & TRUE & TRUE \\
\hline Overall Taste & TRUE & TRUE & FALSE \\
\hline Sweet & FALSE & FALSE & FALSE \\
\hline Salty & FALSE & FALSE & FALSE \\
\hline Sour & TRUE & TRUE & FALSE \\
\hline Bitter & FALSE & TRUE & FALSE \\
\hline
\end{tabular}

olfactory processing and memory consolidation [27, 28, 29, 30]. The hippocampus appears to also play a role in odour information processing [2]. A strong positive relationship between left hippocampal volume and odour identification ability has been observed [2]. In LOAD, significant left hippocampal volume loss was highly correlated with impairments in odour identification ability [2].

As shown in Table 4, HA and AD groups have impaired overall taste abilities, compared to their younger counterparts $(p=0.018$ and $p=2.77 \times 10-4$ for HA and $A D$, respectively). No significant differences were found in overall taste abilities between HA and AD $(p=0.27)$. This is contrary to the recent findings of Steinbach et al. (2010), who showed a reduction in gustatory performance in LOAD and mild-cognitively impaired (MCI) patients, over and beyond that which occurs in normal aging. The lack of significant findings for overall taste in the current study may reflect the low population size of $\mathrm{AD}$ compared to HA groups in this study.

\section{Recognition of the four basic tastes}

A post-hoc, Multiple Comparison after Kruskal-Wallis conducted on the basic taste data collected from each group revealed that identification of sweet and salty taste perception was not greatly impaired in AD or in HA (Table 4). Although differences for sweet identification scores were not significant between the three groups, the spread of data for sweet identification in the three groups is largely

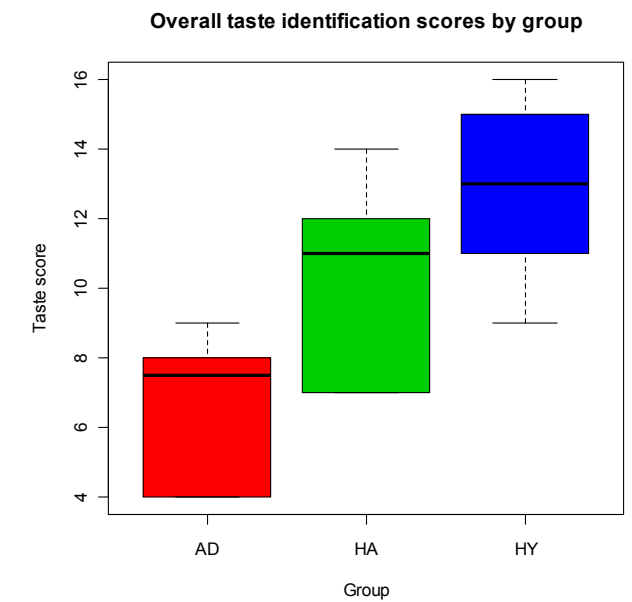

Figure 1: Box-plot displaying smell identification scores (1a) and overall taste scores (1b) in LOAD (AD), healthy aging (HA) and in healthy young (HY) groups. 


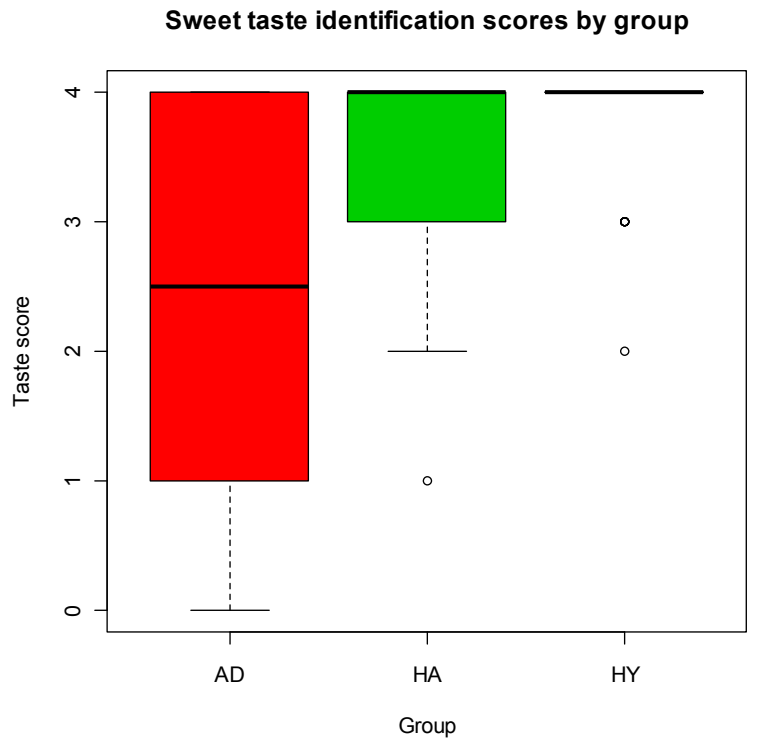

Sour taste identification scores by group

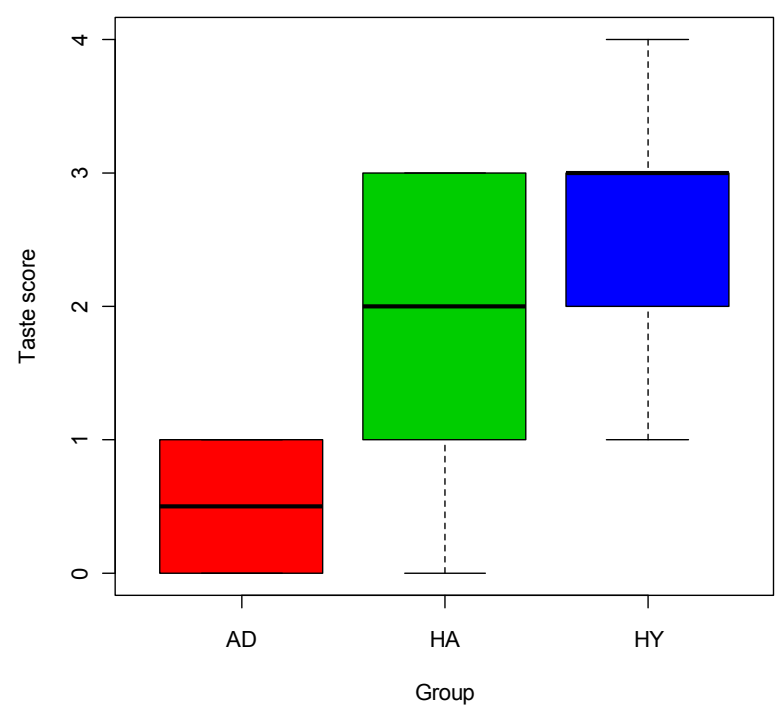

Salty taste identification scores by group

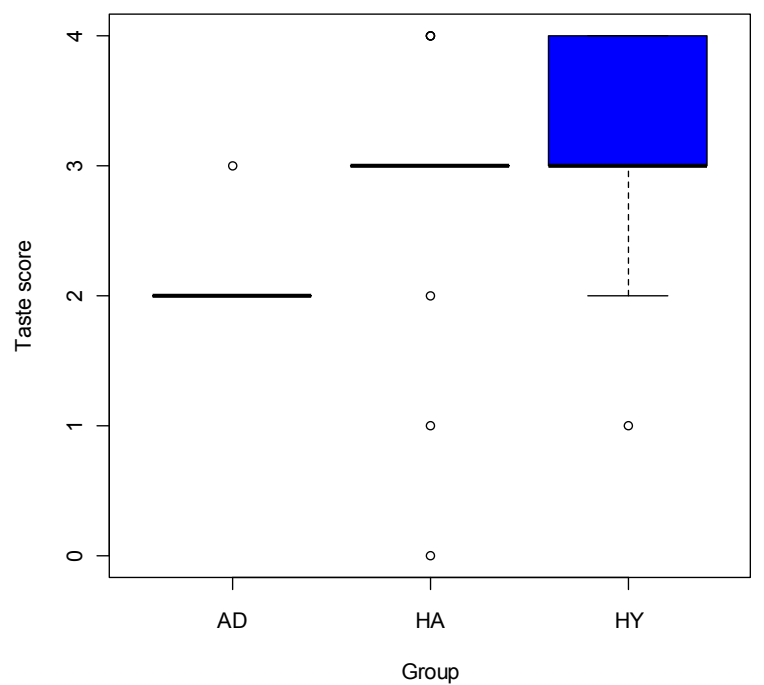

Bitter taste identification scores by group

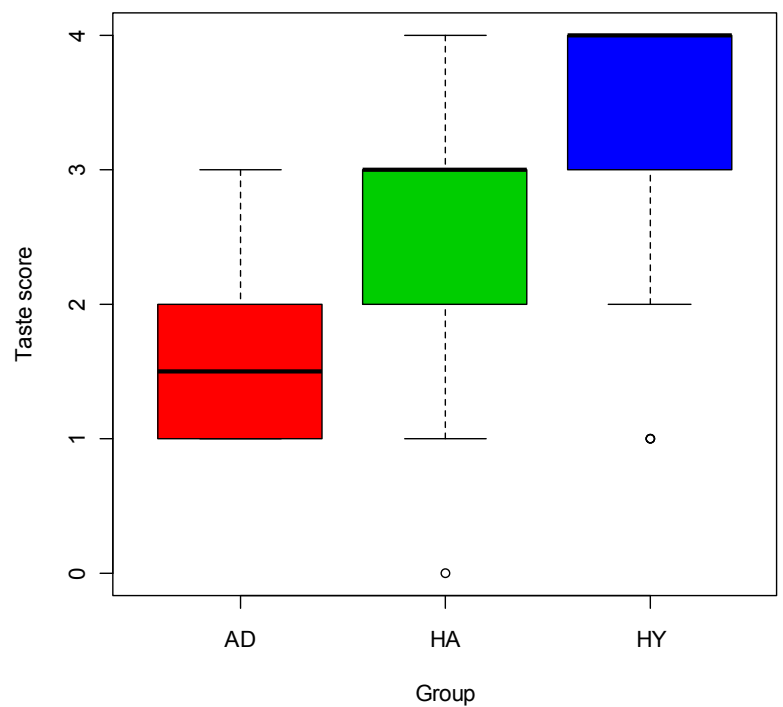

Figure 2: Box-plot displaying sweet (2a), salty (2b), sour (2c) and bitter (2d) identification scores in LOAD (AD), healthy aging (HA) and in healthy young (HY) groups.

dissimilar (Figure 2a). All HY participants scored a four except for a few possible outliers (open circles in Figure 2a). In contrast, the $\mathrm{AD}$ group showed large variations in sweet recognition, with scores ranging from zero to four. Salty identification scores had exceedingly small variability in AD and HA groups (Figure 2b), with the majority of people scoring 2 and 3 , respectively.

Although research showed that perception of the intensity of sweetness and saltiness declines in aging, results of this study showed that sweet and salty identification is still maintained in healthy aging and in those with Alzheimer's disease. This could be important in preventing weight loss and nutritional status impairments. Sweet and slightly salty tastes are palatable to humans and are an indication of caloric food items. Further research should investigate finding the right balance between maintaining the nutritional status of healthy aging and Alzheimer's disease individuals, without the excessive intakes of sweet and salty foods.

There was an aging effect for sour taste recognition. HY individuals were more able to identify sour than HA 
$(\mathrm{p}=0.014)$ and $\mathrm{AD}$ individuals $(\mathrm{p}=3.1 \times 10-4)$ (Table 4). All AD participants achieved a low score of zero or one in their sour recognition test (Figure 2c). However, no significant differences were found in the ability to identify sour between HA and AD groups $(p=0.32)$. This is inconsistent with previous findings, showing AD participants having impaired sour recognition [10]. Medications can alter taste by reducing the function of channels and receptors or producing perceptual distortion of tastes [19]. Some medications have also been reported to enhance certain tastes. Some HA volunteers were taking anti-bacterial medication, which may have the effect of releasing protons and activating ionic taste channels, thereby producing a sour or metallic taste [19].

Bitter recognition abilities for all three groups were contrasted in Figure 2d). AD participants showed bitter impairments when scores were compared to the HY group $(\mathrm{p}=0.012)$ but not when compared to the HA group $(\mathrm{p}=0.71)$. Bitter recognition did not differ across ages in the healthy groups, HY and HA ( $p=0.11$ ). This may be due to the fact that the HA group took significantly more medications than their HY counterparts (Table 1).

Sour and salt detection are mediated through ion channels, specifically by epithelial amiloride-sensitive sodium channel and degenerin (ENaC/DEG). In contrast, sweet and bitter tastes are mediated through transduction signalling mechanisms involving the T1R family of Gprotein coupled receptors (GPCRs). In normal aging, a general taste loss may be occurring due to changes in cell membranes, altering the function of ion channels and membrane receptors [18]. Taste identification impairments in normal aging could also be attributed to reduced activations in the frontal operculum and insula cortex $[3,31]$. These areas of the brain are responsive to taste stimuli and may be responsible for processing information about the identity of a stimulus. There is very limited research in this field and further investigations need to be done on the central decoding processes of taste in young and older adults.

\section{Cognitive ability and its effect on taste and smell}

Correlation coefficient (R) values for smell and taste against neuropsychological tests are shown in Table 5. The inclusion criteria for healthy control participants is an MMSE score of $>23$. MMSE. Scores $<23$ is a warning sign clinicians use to detect possible cognitive impairment [12]. It is possible that these participants are in early, prodromal Alzheimer's disease, where initial brain lesions are occurring but symptoms were not severe enough to be classified as having
Table 5: Correlation coefficient $(R)$ values determined using the Spearman's method for smell and taste recognition abilities, with neuropsychological tests as independent factors.

\begin{tabular}{|l|l|l|l|l|}
\hline & MMSE & Immediate & Delayed & Clock Test \\
\hline Smell test & $0.3827^{* *}$ & $0.4300^{* *}$ & $0.3656^{*}$ & $0.4124^{* *}$ \\
\hline Overall taste & $0.3433^{*}$ & $0.4482^{* *}$ & $0.5220^{* * *}$ & $0.3217^{*}$ \\
\hline Sweet & 0.0777 & 0.1899 & 0.2295 & 0.2397 \\
\hline Salty & 0.1227 & 0.1936 & $0.2997^{*}$ & 0.2684 \\
\hline Sour & $0.4401^{* *}$ & $0.5685^{* * *}$ & $0.5901^{* * *}$ & 0.2750 \\
\hline Bitter & 0.2771 & $0.3376^{*}$ & $0.4280^{* *}$ & 0.2551 \\
\hline
\end{tabular}

probable Alzheimer's disease. The MMSE has poor discrimination to detect demented and non-demented people, when used in isolation [13]. It is also not very sensitive in detecting early-stage LOAD. The Clock-Drawing Test and the CERAD immediate and delayed word recall tests supplemented the MMSE test to assess memory and cognitive skills. The combination of these tests may be able to tap into a broad range of cognitive domains that may be impaired in LOAD. The results collected using the neuropsychological tests were highly dependent upon age and education level (Table 5). Smell and overall taste recognition abilities were strongly and positively correlated to all neuropyschological testing results. When being exposed to a taste or smell stimuli for the first time and to be able to verbally recall that same taste or smell after a period of time, would have required memory consolidation. When doing the smell and taste test, participants consolidated and recalled the smell or taste to obtain the right answer. Similarly, the neuropsychological tests utilized in this experiment assessed all kinds of memory. For example, consolidation and subsequent recall of visual spatial information, motor programming and conceptualization of time would be needed for successful completion of drawing a clock. Thus, it may not be surprising to find similar brain areas, related to memory and recall, activated in both neuropsychological and chemosensory tasks in this study.

Stepwise GLM for screening independent variables most likely to predict health status

We explored 14 variables in this study (age, sex, gender, highest education, MMSE, immediate word recall, delayed word recall, smell, overall taste, sweet, sour, salty, bitter) that may be the most predictive independent variables to model health status in older adults. The stepwise regression started with an AIC of 28. Many variables were eliminated until AIC reached 6, where the most predictive model of health status was determined. Table 6 identifies the

Table 6: Selecting independent variables that most significantly contributed to the variation in health status (ie. presence or absence of LOAD), using stepwise generalized linear model (GLM). The final prediction model for health status is in the form of $Y=B_{1} X_{1}+$ $B_{2} X_{2}+C$ and includes values: 1253.68 for intercept " $C$ ", $-\mathbf{8 2 . 2 1}$ (clock test) for ' $B_{1}$ " and -61.57 (sweet test) for " $B_{2}$ ". The last three steps of the stepwise multiple regression are shown in this table, resulting in the final predictive linear model.

\begin{tabular}{|l|r|r|r|r|r|r|r|r|r|r|r|r|}
\hline \multicolumn{2}{|c|}{} & \multicolumn{2}{|c|}{ Immediate word recall } & & \multicolumn{2}{|c|}{ Delayed word recall } & \multicolumn{3}{|c|}{ Clock test } & \multicolumn{2}{|c|}{ Sweet } \\
\hline & DF & D & AIC & DF & D & AIC & DF & D & AIC & DF & D & AIC \\
\hline Step: & 1 & 0.00 & 8.00 & 1 & 0.00 & 8.00 & 1 & 0.00 & 8.00 & 1 & 4.07 & 12.07 \\
\hline Step: & & & & 1 & 0.00 & 6.00 & 1 & 0.00 & 6.00 & 1 & 4.64 & 10.64 \\
\hline Step: & & & & & & & 1 & 29.21 & 33.21 & 1 & 13.45 & 17.45 \\
\hline
\end{tabular}

$\mathrm{DF}=$ degree freedom; $\mathrm{D}=$ deviance; $\mathrm{AIC}=$ Akaike information criteria 
independent variables most likely to contribute variation to health status. The generalized linear equation was given as follows: $\mathrm{Y}=-82.21 \mathrm{X} 1-61.57 \mathrm{X} 2+1253.68$. Variables $\mathrm{Y}, \mathrm{X} 1$ and $\mathrm{X} 2$ corresponds to health status, clock test scores and sweet recognition abilities, respectively $(\mathrm{DF}=47$; Total Residual $=45$; Null Deviance $=36.17$; Residual Deviance $=$ $9.73 \times 10-9$; AIC: 6 ). There is more strength in GLM than there is with the Kruskal-Wallis or Spearman's correlation analysis. Thus, one could infer more about the relationship between several independent predictor variables and a dependent variable, such as health status. Predictive variables included in the final model that most influenced variations in health status were the clock test and sweet recognition test.

Compared to healthy aging individuals, clock drawing abilities are significantly impaired in LOAD [13]. The clock drawing test is a popular screening test for LOAD and has a high sensitivity to differentiate probable LOAD from normal individuals. The ability to identify sweet, however, should be investigated in further detail in healthy aging and LOAD individuals. Research efforts have identified the molecular mechanisms of sweet detection at the peripheral level but not much is known about the nuclear regions in the brain involved in sweet identification. Some studies describe that in aging, regional deficits on the tongue are more common than whole-mouth taste deficits [18, 32]. In this study, we investigated left and right sides of the tongue, making sure that the tip, lateral and medial left or right sides of the tongue were touching the paper strips. Past investigations have shown that in healthy young and aging adults, the ability to identify sucrose is higher on the tip and mid-lateral regions, than the postero-medial region [32]. Additional experiments should be done to investigate the four basic tastes in certain localized areas of the tongue in LOAD.

\section{CONCLUSION}

This is the first study comparing smell and taste status across ages and early stages of Late-Onset Alzheimer's disease. An age-associated impairment was found for sour and bitter identification. There was a LOAD-related impairment in the ability to identify odours but no such impairments were found for the four basic tastes, when compared to the HA group. However, a predictive model showed that the clock test and sweet recognition performance highly influenced variations in health status. Further research should look at studying smell and taste identification in $\mathrm{HA}$ and $\mathrm{AD}$ individuals, especially in certain localized areas of the tongue. More neuroanatomical research is needed to investigate how the four basic tastes are decoded in higher brain regions. These findings may be important step toward developing methods to in gearing research towards developing methods to maintain the nutritional status of those with LOAD. Furthermore, results may aid in establishing a chemosensory profile for those in pre-clinical stages to enhance current diagnosis criteria of LOAD.

\section{REFERENCES}

1. Brookmeyer, R., Gray, S. and Kawas, C. (1998) Projections of Alzheimer's disease in the united states and the public health impact of delaying disease onset. Am J Public Health. 88, 1337-42.

2. Murphy, C. (2003) Left hippocampal volume loss in Alzheimer's disease is reflected in performance on odour identification: a structural MRI study. Journal of the International Neuropyschological Society. 9, 459471.

3. Murphy, C. (2008) The chemical senses and nutrition in older adults. Journal of Nutrition for the elderly. 27, 247-265.

4. Broggio, E., Pluchon, C., Ingrad, P. and Gil, R. (2001) Taste impairment in Alzheimer's disease. Rev. Neurol. 157, 409-413.

5. Heckmann, J.G., Heckmann, S.M., Lang, C.J.G. and Hummel, T. (2003) Neurological aspects of taste disorders. Neurological Review. 60, 667-671.

6. Yamagishi, M., Takami, S. and Getchell, T.V. (1995) Innervation in human taste buds and its decrease in Alzheimer's disease patients. Acta Otolaryngol (Stockh). 115, 678-684.

7. Gardiner, J., Barton, D., Vanslambrouck, J.M., Braet, F., Hall, D., Marc, J. and Overall, R. (2008) Defects in tongue papillae and taste sensation indicate a problem with neurotrophic support in various neurological diseases. The Neuroscientist. 14, 240-250.

8. Schiffmann, S.S., Clark, C.M. and Warwick, Z.S. (1990) Gustatory and olfactory dysfunction in dementia: not specific to Alzheimer's disease. Neurobiology of Aging. 11, 597-600.

9. Lang, C.J.G., Leuschner, T., Ulrich, K., Stobel, C., Heckmann, J.G. and Hummel, T. (2006) Taste in dementing diseases and parkinsonism. $J$. of Neurological Sciences. 248, 177-184.

10. Steinbach, S., Hundt, W., Vaitl, A., Heinrich, P. and Burger, K. (2010) Taste in mild cognitive impairment and Alzheimer's disease. J. Neurol. 257, 238-246.

11. Campion, D., Dumanchin, C., Hannequin, D., Dubois, B., Belliard, S., Puel, M., Thomas-Anterion, C., Michon, A., Martin, C., Charbonnier, F., Raux, G., Camuzat, A., Penet, C., Mesnage, V., Martinez, M., Clerget-Darpoux, F., Brice, A. and Frebourgl, T. Am. 1999. Early-Onset Autosomal Dominant Alzheimer Disease: Prevalence, Genetic Heterogeneity, and Mutation Spectrum. 65:664-670. 
12. Folstein, M.F., Folstein, S.E. and Mchugh, P.R. (1975) Mini mental state a practical method for grading the cognitive state of patients for the clinician. J. Psychiat. Res. 12, 189-198.

13. Juby, A., Tench, S. and Baker, V. (2002) The value of clock drawing in identifying executive cognitive dysfunction in people with a normal mini-mental state examination score. Canadian Medical Association Journal 167, 859-64.

14. Wicklund, A.H., Johnson, N., Rademaker, A., Weitner, W.B.B. and Weintraub, S. (2006) Word list versus story memory in Alzheimer's disease and frontotemporal dementia. Alzheimer Dis Assoc Disorder. 20, 86-92.

15. Souder, E., O'sullivan, P. and Pechenik, G. (1999) Comparison of scoring criteria for clock drawing test. Journal of Clinical Geropsychology. 5, 139-145.

16. Allison, P.D. (1999) Multiple regression. Thousand Oaks, CA: Pine Forge Press.

17. Berk, R.A. (2003) Regression analysis: A constructive critique. Thousand Oaks, CA: Sage Publications.

18. Boyce, J.R. and Shone, G.R. (2006) Effects of ageing on smell and taste. Postgrad Med J 82, 239-241.

19. Doty, R.L., Shah, M. and Bromley, S.M. (2008) Druginduced taste disorders. Drug Safety 31, 199-215.

20. Chang, W.I., Chung, J.W., Kim, Y.K., Chung, S.C. and Kho, H.S. (2006) The relationship between phenylthiocarbamide (PTC) and 6-n-propylthiouracil (PROP) taste status and taste thresholds for sucrose and quinine. Archives of Oral Biology 51, 427-432.

21. Bartoshuk, L.M., Duffy, V.B. and Miller, I.J. (1994) PTC/PROP tasting: anatomy, psychophysics and sex effects. Physiology \& Behaviour 56, 1165-1171.

22. Henkin, R.I., Velicu, I. (2008) cAMP and cGMP in nasal mucos: relationships to taste and smell dysfunction, gender and age. Clin Invest Med 31, E71E77.

23. Henkin, R.I., Velicu, I. and Papathanassiu, A. (2007) cAMP and cGMP in human parotid saliva: relationships to taste and smell dysfunction, gender and age. The
American Journal of the Medical Sciences 334, 431440.

24. Murphy, C. (1999) Loss of olfactory function in dementing diseases. Physiology \& Behaviour 66, $177-$ 182.

25. Murphy, C. (2002) Olfactory functional testing: sensitivity and specificity for Alzheimer's disease. Drug Development Research 56, 123-131.

26. Thomann, P.A., Santos, V.D., Toro, P., Schonknecht, P., Essig, M. and Schroder, J. (2009) Reduced olfactory bulb and tract volume in early Alzheimer's disease-A MRI study. Neurobiology of Aging 30, 838-841.

27. Braak, E. and Braak, H. (1994) Morphological criteria for the recognition of Alzheimer's disease and the distribution pattern of cortical changes related to this disorder. Neurobiology of Aging 15, 355-356.

28. Braak, E. and Braak, H. (1997) Frequency of stages of Alzheimer-related lesions in different age categories. Neurobiology of Aging. 18, 351-357.

29. Price, J.L., Davis, P.B., Morris, J.C. and White, D.L. (1991) The distribution of tangles, plaques and related immunohistochemical markers in healthy aging and Alzheimer's disease. Neurobiology of Aging 12, 295312.

30. Schiffman, S.S., Graham, B.G., Sattely-Miller, E.A., Zervakis, J. and Welsh-Bohmer, K. (2002) Taste, smell and neuropsychological performance of individuals at familial risk for Alzheimer's disease. Neurobiology of Aging 23, 397-404.

31. Rolls, E.T. 2003. Vision, emotion and memory: From neurophysiology to computation. In cognition and emotion in brain. Selected topics of the International Symposium on limbic and association cortical systems. International Congress Series 1250, 547-573.

32. Nordin, S., Bramerson, A., Bringlov, E., Kobal, G., Hummel, T. and Bende, M. (2007) Substance and tongue-region specific loss in basic taste-quality identification in elderly adults. Eur. Arch. Otorhinolarylngol 264, 285-289. 